\title{
Escape from the Red Queen: an overlooked scenario in coevolutionary studies
}

\author{
Tommy L. F. Leung, Kayla C. King and Justyna Wolinska \\ T. L. F. Leung (tommylfleung@gmail.com), Zoology, School of Environmental and Rural Science, Univ. of New England, Armidale, NSW \\ 2351, Australia. - K. C. King, Dept of Biology, Indiana Univ., 1001 East Third Street, Bloomington, IN 47405-3700, USA. Present address: \\ Inst. of Integrative Biology, Univ. of Liverpool, Crown Street, Liverpool, L69 7ZB, UK. -J. Wolinska, Ludwig-Maximilians-Univ., Dept \\ Biologie II, Evolutionsökologie, Großhaderner Str. 2, DE-82152 Planegg-Martinsried, Germany.
}

\begin{abstract}
Almost all eukaryotic organisms undergo sexual recombination at some stage of their life history. However, strictly asexual organisms should have higher per capita rate of reproduction compared with those that have sex, so the latter must convey some advantage which overrides the reproductive benefit of asexuality. For example, sexual reproduction and recombination may play an important role in allowing organisms to evolutionarily 'keep up' with parasites. Host-parasite coevolution can operate via negative frequency-dependent selection whereby parasite genotypes adapt to infect host genotypes as they become locally common. By producing more genetically diverse offspring with unique genotypes, sexual organisms have an advantage over asexual counterparts. Essentially, sexual hosts are more difficult for coevolving parasites to 'track' over time. This scenario has been named the "Red Queen hypothesis". It refers to a passage in Lewis Carroll's 'Through the Looking Glass' in which the Red Queen tells Alice: 'it takes all the running you can do, to keep in the same place'; this statement resembles the negative frequency-dependent dynamics of host-parasite coevolution.
\end{abstract}

Almost all eukaryotic organisms undergo sexual recombination at some stage of their life history. However, strictly asexual organisms should have higher per capita rate of reproduction compared with those that have sex, so the latter must convey some advantage which overrides the reproductive benefit of asexuality (Maynard Smith 1978, Bell 1982). For example, sexual reproduction and recombination may play an important role in allowing organisms to evolutionarily 'keep up' with parasites. Host-parasite coevolution can operate via negative frequency-dependent selection whereby parasite genotypes adapt to infect host genotypes as they become locally common. By producing more genetically diverse offspring with unique genotypes, sexual organisms have an advantage over asexual counterparts. Essentially, sexual hosts are more difficult for coevolving parasites to 'track' over time (Levin 1975, Jaenike 1978). This scenario has been named the "Red Queen hypothesis" (Jaenike 1978, Hamilton 1980, Bell 1982). It refers to a passage in Lewis Carroll's 'Through the Looking Glass' (1871) in which the Red Queen tells Alice: 'it takes all the running you can do, to keep in the same place'; this statement resembles the negative frequency-dependent dynamics of host-parasite coevolution.

The Red Queen hypothesis may provide an explanation for why sex is so common and asexual reproduction is rare: asexual lineages are more vulnerable to extinction from coevolving parasites (Morran et al. 2011). This hypothesis is difficult to reconcile with the existence of various ancient 'asexual scandals' in nature (Judson and Normark 1996). Such organisms have reproduced exclusively asexually over a considerable period of evolutionary time. They are 'scandalous' (Judson and Normark 1996) because most eukaryotic lineages incorporate some form of sexual genetic recombination at a life-cycle stage, which prevents them from being overwhelmed by harmful parasites coevolved to bypass host defences. The persistence of these 'asexual scandals' indicates that they must have a means of escaping. For example, if hosts can physically disperse away from an infectious environment or have a parasite-free life history phase, these can be effective long-term strategies which allow for the maintenance of asexuality despite the presence of parasites.

We will focus on two recent studies featuring hostparasite systems in which there is empirical evidence of such escape: rotifers (Wilson and Sherman 2010) and microscopic algae (Frada et al. 2008). There are certain theoretical requirements for the 'host escape' scenario to become an evolutionary stable strategy. The host population needs a well-defined metapopulation structure, wherein host migration surpasses parasite migration, and parasite migration is independent of host migration (Ladle et al. 1993, Judson 1995). In essence, hosts can disperse or migrate across space to parasite-free regions or escape across time (Tooby 1982). With the asymmetry in host and parasite migration rates, asexual organisms will not succumb to 
parasites and be able to outcompete sexual conspecifics (Ladle et al. 1993, Judson 1995, 1997).

\section{Empirical evidence of 'host escape'}

\section{Across time and space}

Bdelloid rotifers are a class of freshwater invertebrates with over 350 species. The entire group is known for being 'ancient asexuals' (Judson and Normark 1996), and also for their ability to survive extended periods of desiccation through anhydrobiosis (Tunnacliffe and Lapinski 2003). Wilson and Sherman (2010) tested the hypothesis that the ability of the rotifer Habrotrocha elusa to survive long periods of desiccation, combined with aerial dispersal, allows this invertebrate to escape a deadly fungal parasite, Rotiferophthora angustispora, an organism less tolerant to desiccation. They exposed rotifer colonies to parasite spores in culture dishes which then underwent desiccation over different durations. Colonies exposed to longer period of desiccation had lower levels of parasitism after rehydration. In addition, a wind chamber was used to examine the role of aerial dispersal in allowing desiccated rotifers to escape this parasite. Rotifer colonies that were established by founders blown in from desiccated, infected populations were often parasite-free, whereas those established by founders transferred without desiccation were wiped out by parasites (Wilson and Sherman 2010). Thus, these 'anciently asexual' rotifers are able to escape from their parasite across time (by entering a dormant state) and across space (through wind dispersal).

\section{Across time}

The single-celled, coccolithophore alga Emiliania huxleyi is arguably the most common and widespread photosynthetic plankton in the ocean (Holligan et al. 1983). These algae have a life cycle which alternates between diploid and haploid phases, and they are capable of asexual propagation during both phases (Houdan et al. 2005). The observed algal blooms are successively terminated over multi-annual timescales by outbreaks of a group of viruses, but there is little sign of genotype turnover in the algal population as each successive bloom was dominated by the same genotype (Martinez et al. 2007). Frada et al. (2008) tested the hypothesis that E. huxleyi enters a haploid phase to escape viruses responsible for terminating blooms of diploid cells. Thus, Frada et al. (2008) mimicked a bloom situation by infecting a culture consisting of both cell types. While the diploid cells crashed due to viral infections, individuals in the haploid phase were able to evade the viruses, and consequently, increased in frequency (Frada et al. 2008). Compared with the diploid cells, the modified surface of the haploid cell provides a barrier against virus attachment. Frada et al. (2008) refers to this type of defence as a 'Cheshire Cat' strategy. In 'Alice's Adventures in Wonderland' (Carroll 1865), the Cheshire Cat makes its body invisible so that it becomes impossible to execute. Thus E. huxleyi, much like the Cheshire Cat, escapes its antagonist by being undetectable. Apart from the advantage of avoiding the cost associated with infection, escaping coevolving parasites may have additional benefits, such as reduced investment into immune defence (Rigby et al. 2002, Schmid-Hempel and Ebert 2003). Escapees can thus invest more energy to host reproduction and/or growth. For example, Frada et al. (2008) suggested that because resources are not invested into host defence, the Cheshire Cat strategy may lead to increased productivity and is an advantage in the presence of competitors.

\section{More 'escapees' in nature?}

There are also other planktonic algae that undergo ploidy changes during their life cycle (Lewis 1985, Vaulot et al. 1994) which might lead to similar escape strategies. Although most of the observed life-history shifts in planktonic algae have been attributed to abiotic factors (Sgrosso et al. 2001, Kremp et al. 2009), it has been shown that biotic factors can also play a role (Rengefors et al. 1998, Fistarol et al. 2004). Thyrhaug et al. (2002) showed that, similar to the Cheshire Cat strategy, part of the cell cycle of the prasinophyte algae Pyramimonas orientalis serves as a refuge from attacking viruses. Since the life-history shifts were shown to provide a refuge against parasites in at least two unrelated algae host species, the Cheshire Cat strategy may be more widespread. Unlike Red Queen host-parasite coevolution which operates via negative frequency-dependent selection, the cycle of susceptibility and resistance associated with ploidy changes appears to result from the phenotypic plasticity of the same reoccurring host genotype(s) (Thyrhaug et al. 2003). Being susceptible only during certain stages of the life-cycle may help phytoplankton populations recover from viral outbreaks. Indeed, the same dominant host genotype of coccolithophore algae continues to reappear over each cycle (Martinez et al. 2007). However, this type of long term dominance by a single genotype is not typical among eukaryotic phytoplankton. For example, Rynearson and Armbrust (2005) identified almost 500 different clonal lineages during a bloom of the diatom Ditylum brightwelli with evidence of past sexual reproduction and/ or recombination events. De Bruin et al. (2004) also found great genetic variation in the population of the diatom Asterionella formosa. In that system, the coevolutionary dynamics of $A$. formosa with its chytrid fungus parasite (Zygorbizidium planktonicum) follow that expected under the Red Queen hypothesis (Ibelings et al. 2004) because there is genotypic specificity in the host-parasite interactions (De Bruin et al. 2004). Thus, although the Cheshire Cat strategy has evolved in E. huxleyi and other algal lineages, this is not typical among all species of eukaryotic phytoplankton.

As is the case for bdelloid rotifers (Wilson and Sherman 2010), other desiccation-tolerant organisms might also be able to escape from coevolving parasites. For example, water and soil dwelling microscopic tardigrades, and some nematodes, are capable of surviving complete desiccation (reviewed by Alpert 2006) and are commonly infected by virulent fungal parasites (Richards 1970, Barron 1978, Jansson et al. 1984). However, the potentially adaptive role of tardigrade or nematode desiccation in the hostparasite coevolutionary race is unknown. Although such escapees would experience less parasite-mediated selection, there may be significant costs associated with desiccation. For example, desiccation-tolerant organisms often have 
certain physiological constraints on their size and growth, reducing their productivity and competitive ability (Alpert 2006). Unlike rotifers, other animals capable of anhydrobiosis might escape their parasites across time, but their ability to escape over space is limited at best. For example, nematodes rarely survive aerial dispersal during anhydrobiosis (Nkem et al. 2006). In addition, although wind dispersal has been documented in tardigrades from the Antarctic region (Nkem et al. 2006), most tardigrade species enter anhydrobiosis amidst moss cushions which limit their exposure to air currents (Wright 1991). Thus, the combination of desiccation tolerance and high mobility make bdelloid rotifers unique (Wilson and Sherman 2010).

Propagules or eggs might also act as an avenue for temporal and spatial escape, particularly if they are desiccationtolerant. Plant seeds have been specifically suggested to serve such a role (Richards 1986, Burt and Bell 1991), and Ladle et al. (1993) proposed that the green algae Protococcus, which reproduces via vegetative spores, might also be an escapee. The dormant eggs of some crustaceans can also tolerate long periods of desiccation (Clegg 2005). Indeed, Ladle et al. (1993) suggested asexual brine shrimps with their desiccation-resistant eggs may be escapees from parasites that cannot otherwise survive desiccation. Moreover, there is field evidence that exposure to some parasites triggers dormant egg production in crustacean waterfleas (Daphnia) (Mitchell et al. 2004), and periods of diapause by resting eggs lead to a decline in microsporidian parasite prevalence (Lass and Ebert 2006). While propagules can play a role in temporal escape, their potential for spatial escape obviously varies depending on the dispersal capability of the organism. Wind dispersal seems to play a role in the aerial transportation of brine shrimp eggs (Brendonck and Riddoch 1999), though the distances achieved by the eggs through wind dispersal are not as pronounced as that reached by rotifer 'tuns' which can cover over $80 \mathrm{~km}$ (Jenkins and Underwood 1998).

\section{Parasite response to host escape}

Do the parasites of escapees have any adaptations for keeping up with the host? Coevolution may still occur, but at a different pace to Red Queen coevolution. The disappearance of the host population and the lack of investment into parasite resistance mechanisms may lead to differential selection pressure on certain parasite traits, such as virulence (Sasaki and Godfray 1999). Coevolutionary cycles may progress from high parasite virulence and host investment into resistance mechanisms to reduced virulence once hosts forego costly resistance (e.g. due to escape) (Sasaki and Godfray 1999). This relationship between virulence and host defence, however, may only be maintained as long as the parasites have high dispersal rates and occur in multiple genotype infections (Gandon 1998).

Survival of parasite propagules in the external environment would be an important trait to maintain when hosts are unavailable. A strong positive correlation has been demonstrated between parasite virulence and the durability of parasite propagules in the environment (Walther and Ewald 2004), although there are contrasting examples (Bonhoeffer et al. 1996). Also known as the "curse of the pharaoh' (Bonhoeffer et al. 1996, Gandon 1998), the relationship between parasite virulence and longevity is named after the incident during which Lord Carnarvon mysteriously died after entering the tomb of Tutankhamen. His death may have been caused by a highly virulent, longlived pathogen.

\section{Conclusions}

Life-history stages which could facilitate a host's escape from coevolving parasites are common in a wide range of taxonomic groups. The mechanisms for escape might have either evolved as a direct response to parasitism or as byproducts of other adaptations. While the rotifer's escape mechanism involves desiccation and the temporal and spatial avoidance of parasites, organisms exhibiting the Cheshire Cat strategy remain in sympatry with their parasites. More examples may exist among organisms with similar lifehistory traits to rotifers and E. huxleyi phytoplankton. These traits include obligate asexuality, dominance by a single or a few genotypes within a given population, life stages protected from infection, and extended dispersal capabilities. While not one escapee taxon would exhibit all those suggested traits, escapees seem to all have a low or no reliance on sexual recombination.

The escapees complement the mounting evidence in favour of the Red Queen hypothesis (Lively et al. 1990, Fischer and Schmid-Hempel 2005, Jokela et al. 2009, King et al. 2009, Morran et al. 2011) as they indicate that asexual reproduction is beneficial and can persist only when coevolving parasites are not a threat. The next step may be to investigate whether sexual recombination occurs less often in potential escapee taxa compared with related taxa which do not exhibit ecological escape and examine how this is affected by coevolving parasites. Studying the link between asexuality and ecological escape from parasites is particularly applicable to cyclical parthenogens. Moreover, future field studies may involve testing whether the lack of recombination in hosts has reciprocally caused the parasites to abandon sex. Finally, since periods of escape can interrupt or halt the coevolutionary arms race, it may prove insightful to follow changes in genotype frequencies within the populations of both hosts and parasites and compare them to the pattern of coevolution with systems without periodic episodes of escape.

Finally, an experimental approach should also be used to investigate escape strategies. It will be worth testing the Cheshire cat strategy further and look at how host phenotypic plasticity in susceptibility to coevolved parasites can affect antagonistic coevolutionary dynamics. For example, this can be achieved with a long term mesocosm algal cultures such as those conducted by Thyraug et al. (2003), but combined with elements of time-shift experiments. One could do this by introducing batches of viruses obtained from different period of viral lysis to the duplicate cultures of the same algal genotype, and quantifying host susceptibility and time to recovery. Another experiment is to compare the infection success of fungal parasites on rotifers which have undergone a series of desiccation and rehydration with those that have undergone fewer. In summary, 
we can gain further insight into what factors drive antagonistic coevolution by investigating the temporal pattern of host-parasite population genetics, as well as by performing laboratory-based, multi-generational, and/or time-shift infection experiments with coevolving parasites and hosts that occasionally escape.

Acknowledgements - The authors would like to thank Prof. Curt Lively and for comments on an earlier draft.

\section{References}

Alpert, P. 2006. Constraints of tolerance: why are desiccationtolerant organisms so small or rare? - J. Exp. Biol. 209: 1575-1584.

Barron, G. L. 1978. Nematophagous fungi: endoparasites of Rhabditis terricola. - Microbiol. Ecol. 4: 157-163.

Bell, G. 1982. The masterpiece of nature. The evolution and genetics of sexuality. - Univ. of California Press.

Bonhoeffer, S. et al. 1996. The curse of the pharaoh: the evolution of virulence in pathogens with long living propagules. - Proc. R. Soc. B 263: 715-721.

Brendonck, L. and Riddoch, B. J. 1999. Wind-borne shortrange egg dispersal in anostracans (Crustacea: Branchiopoda). - Biol. J. Linn. Soc. 67: 87-95.

Burt, A. and Bell, G. 1991. Seed reproduction is associated with a transient escape from parasite damage in American beech. - Oikos 61: 145-148.

Carroll, L. 1865. Alice's Adventures in Wonderland. - MacMillan and Co.

Carroll, L. 1871. Through the looking glass and what Alice found there. - MacMillan and Co.

Clegg, J. S. 2005. Desiccation tolerance in encysted embryos of the animal extremophile, Artemia. - Integr. Com. Biol. 45: $715-724$

De Bruin, A. et al. 2004. Genetic variation in Asterionella formosa (Bacillariophyceae): is it linked to frequent epidemics of hostspecific parasitic fungi? - J. Phycol. 40: 823-830.

Fischer, O. and Schmid-Hempel, P. 2005. Selection by parasites may increase host recombination frequency. - Biol. Lett. 1: 193-195.

Fistarol, G. O. et al. 2004. Temporary cyst formation in phytoplankton: a response to allelopathic competitors? - Environ. Microbiol. 6: 791-798.

Frada, M. et al. 2008. The 'Cheshire Cat' escape strategy of the coccolithophore Emiliania huxleyi in response to viral infection. - Proc. Natl Acad. Sci. USA 105: 15944-15949.

Gandon, S. 1998. The curse of the pharaoh hypothesis. - Proc. R. Soc. B 265: 1545-1552.

Hamilton, W. D. 1980. Sex versus non-sex versus parasite. - Oikos 35: 282-290.

Holligan, P. M. et al. 1983. Satellite and ship studies of coccolithophore production along a continental shelf edge. - Nature 304: 339-342.

Houdan, A. et al. 2005. Comparison of photosynthetic responses in diploid and haploid life-cycle phases of Emiliania huxleyi (Prymnesiophyceae). - Mar. Ecol. Prog. Ser. 292: 139-146.

Ibelings, B. W. et al. 2004. Host-parasite interactions between freshwater phytoplankton and chytrid fungi (Chytridiomycota). - J. Phycol. 40: 437-453.

Jaenike, J. 1978. A hypothesis to account for the maintenance of sex within populations. - Evol. Theory 3: 191-194.

Jansson, H. B. et al. 1984. Life cycle of the endoparasitic nematophagous fundus Meria coniospora: a light microscopic and electron microscopic study. - J. Microbiol. 50: 321-328.
Jenkins, D. G. and Underwood, M. O. 1998. Zooplankton may not disperse readily in wind, rain or waterfowl. - Hydrobiologia 387: 15-21.

Jokela, J. et al. 2009. The maintenance of sex, clonal dynamics, and host-parasite coevolution in a mixed population of sexual and asexual snails. - Am. Nat. 174: S43-S53.

Judson, O. P. 1995. Preserving genes - a model of the maintenance of genetic variation in a metapopulation under frequency dependent selection. - Genet. Res. 65: 175-191.

Judson, O. P. 1997. A model of asexuality and clonal diversity: cloning the red queen. $-\mathrm{J}$. Theor. Biol. 186: 33-40.

Judson, O. P. and Normark, B. B. 1996. Ancient asexual scandals. - Trends Ecol. Evol. 11: A41-A46.

King, K. C. et al. 2009. The geographic mosaic of sex and the Red Queen. - Curr. Biol. 19: 1438-1441.

Kremp, A. et al. 2009. Species-specific encystment patterns in three Baltic cold-water dinoflagellates: the role of multiple cues in resting cyst formation. - Limnol. Oceanogr. 54: 1125-1138.

Ladle, R. J. et al. 1993. Coevolutionary dynamics of sex in a metapopulation - escaping the Red Queen. - Proc. R. Soc. B 253: $155-160$.

Lass, S. and Ebert, D. 2006. Apparent seasonality of parasite dynamics: analysis of cyclic prevalence patterns. - Proc. R. Soc. B 273: 199-206.

Levin, D. A. 1975. Pest pressure and recombination systems in plants. - Am. Nat. 109: 437-457.

Lewis, W. M. 1985. Nutrient scarcity as an evolutionary cause of haploidy. - Am. Nat. 125: 692-701.

Lively, C. M. et al. 1990. Red Queen hypothesis supported by parasitism in sexual and clonal fish. - Nature 344: 864-866.

Martinez, J. M. et al. 2007. Molecular dynamics of Emiliania huxleyi and cooccurring viruses during two separate mesocosm studies. - Appl. Environ. Microbiol. 73: 554-562.

Maynard Smith, J. 1978. The evolution of sex. - Cambridge Univ. Press.

Mitchell, S. E. et al. 2004. The effect of a pathogen epidemic on the genetic structure and reproductive strategy of the crustacean Daphnia magna. - Ecol. Lett. 7: 848-858.

Morran, L. T. et al. 2011. Running with the Red Queen: host-parasite coevolution selects for biparental sex. - Science 333: 216-218.

Nkem, J. N. et al. 2006. Wind dispersal of soil invertebrates in the McMurdo Dry valleys, Antarctica. - Polar Biol. 29: 346-352.

Rengefors, K. et al. 1998. Algal cyst dormancy: a temporal escape from herbivory. - Proc. R. Soc. B 265: 1353-1358.

Richards, A. J. 1986. Plant breeding systems. - Allen and Unwin.

Richards, M. J. 1970. Ballocephala verrucospora sp. nov., parasitizing tardigrades. - Trans. Brit. Mycol. Soc. 55: 307-309.

Rigby, M. C. et al. 2002. Why should parasite resistance be costly? - Trends Parasitol. 18: 116-120.

Rynearson, T. A. and Armbrust, E. V. 2005. Maintenance of clonal diversity during a spring bloom of the centric diatom Ditylum brightwellii. - Mol. Ecol. 14: 1631-1640.

Sasaki, A. and Godfray, H. C. J. 1999. A model for the coevolution of resistance and virulence in coupled host-parasitoid interactions. - Proc. R. Soc. B 266: 455-463.

Schmid-Hempel, P. and Ebert, D. 2003. On the evolutionary ecology of specific immune defence. - Trends Ecol. Evol. 18: 27-32.

Sgrosso, S. et al. 2001. Temperature and daylength regulate encystment in calcareous cyst-forming dinoflagellates. - Mar. Ecol. Prog. Ser. 211: 77-87.

Thyrhaug, R. et al. 2002. Cell cycle dependent virus production in marine phytoplankton. - J. Phycol. 38: 338-343.

Thyrhaug, R. et al. 2003. Stable coexistence in marine algal hostvirus systems. - Mar. Ecol. Prog. Ser. 254: 27-35. 
Tooby, J. 1982. Pathogens, polymorphism, and the evolution of sex. - J. Theor. Biol. 97: 557-576.

Tunnacliffe, A. and Lapinski, J. 2003. Resurrecting Van Leeuwenhoek's rotifers: a reappraisal of the role of disaccharides in anhydrobiosis. - Phil. Trans. R. Soc. B 358: 1755-1771.

Vaulot, D. et al. 1994. Morphology, ploidy, pigment composition, and genome size of cultured strains of Phaeocystis (Prymnesiophyceae). - J. Phycol. 30: 1022-1035.
Walther, B. A. and Ewald, P. W. 2004. Pathogen survival in the external environment and the evolution of virulence. - Biol. Rev. 79: 849-869.

Wilson, C. G. and Sherman, P. W. 2010. Anciently asexual bdelloid rotifers escape lethal fungal parasites by drying up and blowing away. - Science 327: 574-576.

Wright, J. C. 1991. The significance of four xeric parameters in the ecology of terrastrail tardigrada. - J. Zool. 224: 59-77. 